\title{
Tiling Polygons with Lattice Triangles
}

\author{
Steve Butler • Fan Chung • Ron Graham • \\ Miklós Laczkovich
}

Received: 20 July 2009 / Revised: 26 January 2010 / Accepted: 26 January 2010 /

Published online: 12 February 2010

(C) Springer Science+Business Media, LLC 2010

\begin{abstract}
Given a simple polygon with rational coordinates having one vertex at the origin and an adjacent vertex on the $x$-axis, we look at the problem of the location of the vertices for a tiling of the polygon using lattice triangles (i.e., triangles which are congruent to a triangle with the coordinates of the vertices being integer). We show that the coordinates of the vertices in any tiling are rationals with the possible denominators odd numbers dependent on the cotangents of the angles in the triangles.
\end{abstract}

Keywords Tiling $\cdot$ Lattice triangles $\cdot$ Rational coordinates $\cdot$ Stomachion

\section{Introduction}

We consider the problem of tiling with triangles a simple polygon with one vertex of the polygon at the origin and an adjacent vertex on the $x$-axis. We will restrict ourselves to the case when the triangles for the tiling consist of congruent copies of $\Delta_{1}, \Delta_{2}, \ldots, \Delta_{k}$ where each $\Delta_{i}$ is a lattice triangle, i.e., a triangle that can be placed

S. Butler was supported by an NSF postdoctoral fellowship.

\section{S. Butler}

University of California, Los Angeles, Los Angeles, CA, USA

e-mail: butler@math.ucla.edu

F. Chung $\cdot$ R. Graham ( $₫)$

University of California, San Diego, San Diego, CA, USA

e-mail: graham@ucsd.edu

F. Chung

e-mail: fan@ucsd.edu

M. Laczkovich

Eötvös Loránd University, Budapest, Hungary

e-mail: laczk@cs.elte.hu 
in the plane so that the coordinates of the vertices are all integer. A simple example of these triangles are right triangles with integer lengths $a_{i}, b_{i}$ for the two legs, in this special case we will refer to these as an $\left(a_{i}, b_{i}\right)$ triangle.

Previous papers [2-6] have looked at tiling polygons using similar triangles, i.e., where we start with a collection of triangles and are allowed to scale the triangles when placing them. In this paper we do not allow for scaling but rather assume that all triangles are congruent to one of several specified lattice triangles in our tiling. Our prohibition of scaling is not so restrictive since it is easy to see that we can combine $j^{2}$ copies of triangle $\Delta_{i}$ to make a scaled version of $\Delta_{i}$ where each side has been scaled by $j$.

We will show in Sect. 2 that the coordinates of the vertices in our tiling are rational numbers with the possible denominators odd numbers dependent on the cotangents of the $\Delta_{i}$. In Sect. 3 we give a construction which shows how it is possible to achieve large terms in the denominators for some internal vertices in a packing of a large square using right triangles $\left(a_{i}, b_{i}\right)$. Finally we give some concluding remarks.

\section{Possible Vertex Locations}

We first start by showing that the coordinates of the vertices in a tiling of a polygon must be of a restricted form. We first observe that the coordinates of all the vertices will be rational. To see this we need the following theorem.

Theorem 1 (Laczkovich [2]) If a simple polygon $P$ is tiled by triangles $\Delta_{1}, \ldots, \Delta_{n}$, then the coordinates of the vertices of each $\Delta_{j}$ belong to the field generated by the coordinates of the vertices of $P$ and the cotangents of the angles of the triangles $\Delta_{j}$.

It is easy to check that a lattice triangle has all cotangents rational (in fact a triangle has all cotangents rational if and only if it is similar to a lattice triangle). So by an application of the above theorem if we pack our polygon with lattice triangles then we must have only rational coordinates. But now we show that we can say even more about the denominators of these rational numbers.

Theorem 2 Let $P$ be a simple polygon in the plane such that the coordinates of the vertices of $P$ are rational, and one of the vertices of $P$ is the origin with an adjacent vertex on the $x$-axis. Suppose that $P$ is dissected into congruent copies of lattice triangles $\Delta_{1}, \Delta_{2}, \ldots, \Delta_{k}$. Let $\alpha_{1}, \alpha_{2}, \ldots, \alpha_{3 k}$ denote the angles of the triangles $\Delta_{i}$ and let $\cot \alpha_{j}=a_{j} / b_{j}$ with coprime integers $a_{j}, b_{j}$ for every $j=1,2, \ldots, 3 k$. Then the coordinates of each vertex in the tiling are of the form $p / \prod_{j=1}^{3 k}\left(a_{j}^{2}+b_{j}^{2}\right)^{n_{j}}$, where $p$ and $n_{1}, n_{2}, \ldots, n_{3 k}$ are integers.

The proof will make use of the following lemma.

Lemma 3 Let $\theta= \pm t_{1} \alpha_{1} \pm t_{2} \alpha_{2} \pm \cdots \pm t_{3 k} \alpha_{3 k}$ with $t_{i}$ nonnegative integers. Then for some integers $u, v, r, s$, with $r$ and $s$ square-free, $\cos \theta=u \sqrt{r} / \prod_{i=1}^{3 k}\left(a_{i}^{2}+b_{i}^{2}\right)^{t_{i}}$ and $\sin \theta=v \sqrt{s} / \prod_{i=1}^{3 k}\left(a_{i}^{2}+b_{i}^{2}\right)^{t_{i}}$. 
This follows since

$$
\left|\sin \alpha_{j}\right|=\frac{1}{\sqrt{1+\cot ^{2} \alpha_{j}}}=\frac{\left|b_{j}\right|}{\sqrt{a_{j}^{2}+b_{j}^{2}}} \quad \text { and } \quad\left|\cos \alpha_{j}\right|=\frac{1}{\sqrt{1+\tan ^{2} \alpha_{j}}}=\frac{\left|a_{j}\right|}{\sqrt{a_{j}^{2}+b_{j}^{2}}} .
$$

An easy induction now shows for $t_{i} \geq 0$ that $\cos \left(t_{i} \alpha_{i}\right)=u_{i} /\left(a_{i}^{2}+b_{i}^{2}\right)^{t_{i} / 2}$ (similarly for $\sin \left(t_{i} \alpha_{i}\right)$ ) and then applying the sum rule for trigonometric functions and rationalizing gives the result.

Proof of Theorem 2 By Theorem 1 we know that all the vertices have rational coordinates and so it remains to show that the rational coordinates are all of the correct form.

Looking at any vertex of the tiling that is not a vertex of $P$ we see that there must always be an edge of our tiling which goes from the vertex either to the left or straight down. So starting at any internal vertex we continue taking such an edge until we get to a vertex of the polygon (this process must stop after finitely many steps because it is impossible to visit any vertex twice and there are only finitely many vertices). If we are not at $(0,0)$ then we can move along the edges of $P$ to get to $(0,0)$. In particular, we can get from the origin to any vertex in the tiling by moving along edges of triangles in the tiling.

For a vertex $(x, y)$ in the tiling fix a path that goes from the origin $(0,0)$ to $(x, y)$ that moves along edges in the tiling. We show by induction that at each vertex in the path we have coordinates of the desired form and so in particular the terminal vertex $(x, y)$ is also of the desired form. We trivially have that $(0,0)$ is of the desired form. Now suppose that $\left(x_{i}, y_{i}\right)$ is a point on the path with rational coordinates and that $\left(x_{i+1}, y_{i+1}\right)$ is the next point on the path and that the distance between them is $\sqrt{a}$ for some integer $a$ (which holds since all triangles are congruent to lattice triangles). We first note that the line segment joining them will form an angle with the $x$-axis of the form

$$
\theta=s_{1} \alpha_{1}+s_{2} \alpha_{2}+\cdots+s_{3 k} \alpha_{3 k}
$$

for some appropriate choice of $s_{i}$. This follows since the initial angle as well as the angle between any two consecutive line segments is composed of some combination of angles of triangles in the tiling. So applying Lemma 3 we have

$$
\begin{aligned}
x_{i+1} & =x_{i}+\sqrt{a} \cos \theta \\
& =x_{i}+\frac{u \sqrt{a r}}{\prod_{i=1}^{3 k}\left(a_{i}^{2}+b_{i}^{2}\right)^{s_{i}}}, \\
y_{i+1} & =y_{i}+\sqrt{a} \sin \theta \\
& =y_{i}+\frac{v \sqrt{a s}}{\prod_{i=1}^{3 k}\left(a_{i}^{2}+b_{i}^{2}\right)^{s_{i}}} .
\end{aligned}
$$

Since both $x_{i}$ and $x_{i+1}$ are rational we must have that $\sqrt{a r}$ is an integer, similarly since $y_{i}$ and $y_{i+1}$ are rational we must have $\sqrt{a s}$ is an integer. By induction since 
$\left(x_{i}, y_{i}\right)$ is of the desired form we also have that $\left(x_{i+1}, y_{i+1}\right)$ is of the desired form, concluding the proof.

This shows that the denominators of the coordinates are dependent on the cotangents. Since no prime of the form $4 k+3$ can divide $a^{2}+b^{2}$ for $a, b$ coprime integers we see that we will never have a prime of that form in the denominator. We can actually show a little more.

Theorem 4 Let $P$ be a simple polygon in the plane such that the coordinates of the vertices of $P$ are rational and one of the vertices of $P$ is the origin. Suppose that $P$ is dissected into lattice triangles. Then the denominators of the coordinates of the vertices are products of primes of the form $4 k+1$.

Proof By Theorem 1 we know that the coordinates of the vertices are rational and so we only need to show that the terms in the denominator are of the correct form.

As in Theorem 2 we can find a path from $(0,0)$ to any vertex moving along edges of the triangles in the dissection. We now proceed by induction along the vertices in the path. Clearly it holds for $(0,0)$. Suppose that it now holds for point $\left(x_{i}, y_{i}\right)$ on the path and consider the point $\left(x_{i+1}, y_{i+1}\right)$. Since the distance between these two is the side of a lattice triangle they are distance $\sqrt{a}$ apart with $a$ an integer.

It suffices to show that $x_{i+1}-x_{i}$ and $y_{i+1}-y_{i}$ both have denominators which are products of primes of the form $4 k+1$. Let $p / q=x_{i+1}-x_{i}$ with $p$ and $q \neq 0$ coprime integers and $r=y_{i+1}-y_{i}$ rational. Then $(p / q)^{2}+r^{2}=a$. Since $p^{2}+(r q)^{2}=a q^{2}$ it follows that $s=r q$ is an integer. Let $p=p_{1} d$ and $s=s_{1} d$ where $d$ is the greatest common divisor of $p$ and $s$. Then $d$ and $q$ are coprime and thus if $t$ is a prime divisor of $q$, then $t$ divides $p_{1}^{2}+s_{1}^{2}$. Since $p_{1}$ and $s_{1}$ are coprime, this implies that $t$ is a prime of the form $4 k+1$ or $t=2$. But the latter is impossible. Indeed, if $t=2$ then $q$ is even, then $p$ is odd, then $s$ is odd, then $p^{2}+s^{2}$ is of the form $4 k+2$, while $a q^{2}$ is divisible by 4 , a contradiction.

\section{Constructing Vertices with Large Denominators}

We have seen that in our tiling with lattice triangles when the coordinates of vertices are rational the denominators must be of a restricted form. In this section we consider the special case when the triangles $\Delta_{i}=\left(a_{i}, b_{i}\right)$ are right triangles (recall that $a_{i}, b_{i}$ are integers which are the length of the legs), and show how one could build a large denominator using congruent copies of these triangles. We will let $\beta_{i}$ be the smallest angle in the triangle $\Delta_{i}$.

Looking at the proof in Theorem 2 we see that the difference between $x_{i}$ and $x_{i+1}$ is of the form $\sqrt{a} \cos \theta$. So if we want to get a large denominator for a vertex then we should get $\cos \theta$ to have a large denominator. Again looking at Lemma 3 we see that to achieve this we want to form an edge which has an angle of the form

$$
\theta=s_{1} \alpha_{1}+s_{2} \alpha_{2}+\cdots+s_{3 k} \alpha_{3 k}
$$


Fig. 1 The basic construction with $(a, b)$ triangles and $a<b$

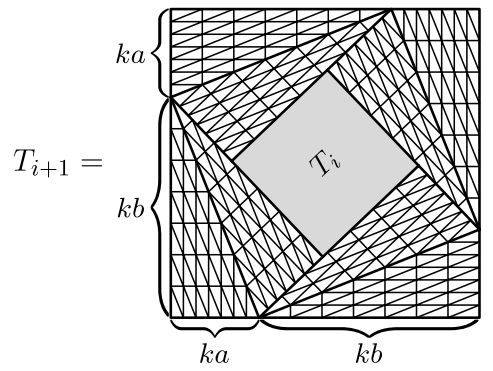

with the $s_{i}$ large. We show how to form a packing of some $[0, N] \times[0, N]$ square ( $N$ to be chosen later) which has an edge in the tiling with slope

$$
\theta=2 s_{1} \beta_{1}+2 s_{2} \beta_{2}+\cdots+2 s_{k} \beta_{k}
$$

and the $s_{i} \geq 0$ arbitrarily large (we also assume $\beta_{i} \neq \pi / 4$ ).

(In general we do not need to have all of the coefficients of the angles be even, for instance for $(3,4)$ triangles where the hypotenuse is integer we can have any coefficient. It is also possible to combine the angles on two different lattice triangles (see Fig. 5 for an example). For our purpose in constructing a large denominator what we have is sufficient.)

The basic construction is shown in Fig. 1. Namely we build a packing of a square one "layer" at a time. Given a square that we want to place in the middle, the idea is to "blow up" triangle $(a, b)$ to triangle $(k a, k b)$ where $k$ is large enough so that we can place the square inside the middle region. Note that the dimensions of the square in the middle is $k(b-a) \times k(b-a)$ and in the worst case scenario we can choose $k$ to be the side length of $T_{i}$ and then pack $(b-a)^{2}$ copies in the middle. After packing $T_{i}$ inside of $T_{i+1}$ we see that the angle of every edge in $T_{i}$ now has an angle of $2 \alpha=2 \arctan (a / b)$ added to it.

So to construct an angle of $\theta$ we find a packing of some square, call it $T_{0}$. We then apply this process $s_{1}$ times using the $\left(a_{1}, b_{1}\right)$ triangles, then $s_{2}$ times using the $\left(a_{2}, b_{2}\right)$ triangles, $\ldots$, and finally $s_{k}$ times using the $\left(a_{k}, b_{k}\right)$ triangles.

An example of this construction is shown in Fig. 2 where we apply the procedure 3 times using $(1,2)$ triangles. The initial packing of $[0,2] \times[0,2]$ has all vertices at integer coordinates. The second generation is a packing of $[0,6] \times[0,6]$ and has all vertices with integer $/ 5$ coordinates including a vertex located at $(16 / 5,8 / 5)$. The third generation is a packing of $[0,18] \times[0,18]$ and has all vertices with integer $/ 25$ coordinates including a vertex located at $(256 / 25,208 / 25)$. The fourth generation is a packing of $[0,54] \times[0,54]$ and has all vertices with integer/ 125 coordinates including a vertex located at $(3536 / 125,3448 / 125)$.

\section{Concluding Remarks}

Looking at the proof of Theorem 2 we see that the coordinates of the vertices can be written in the form $\left(\sum \cos \theta_{j} e_{j}, \sum \sin \theta_{j} e_{j}\right)$ where $\cos \theta_{j}, \sin \theta_{j} \in \mathbb{Z}\left[\cos \alpha_{i}, \sin \alpha_{i}\right.$ : 

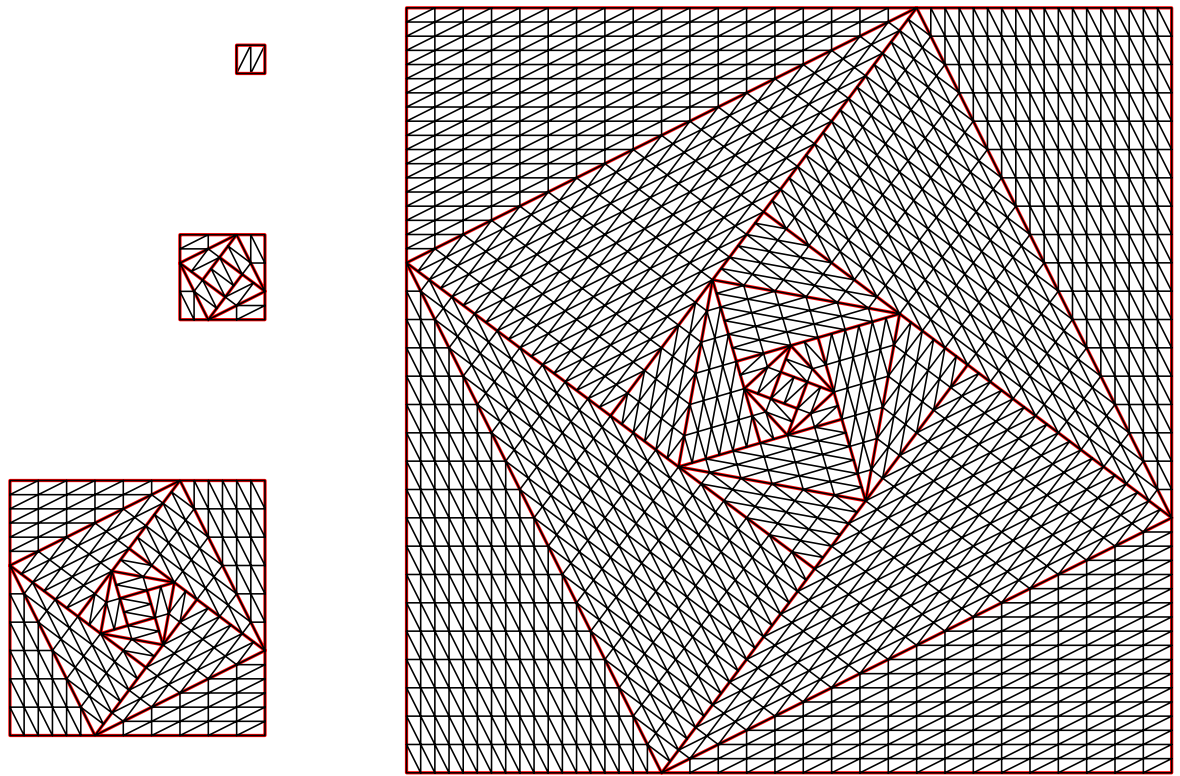

Fig. 2 An example of the iterative construction with $(1,2)$ triangles

Fig. 3 Archimedes' Stomachion

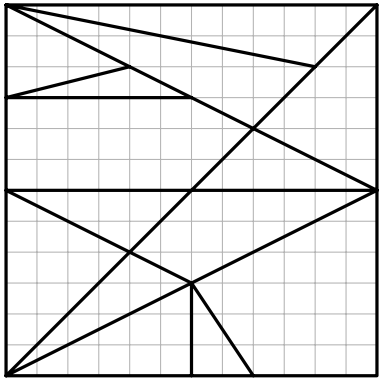

$i=1,2, \ldots, 3 k]$ and $e_{j}$ comes from the set of edge lengths. In other words the coordinates lie in the $R$-module where our ring is generated by the sines and cosines of the angles of the triangles and the abelian group is generated by the edge lengths of the triangles. This is more restrictive than Theorem 1 where the vertices are in the field generated by the cotangents of the angles.

We can also consider tilings where instead of using congruent lattice triangles we use congruent polygons. For example, Archimedes' Stomachion is a subdivision of the square into fourteen polygonal pieces (see Fig. 3). It is known [1] that there are 268 ways to arrange the pieces of the Stomachion to tile a square. Further if we put any of these arrangements of the Stomachion on $[0,12] \times[0,12]$ each vertex will be located at integer coordinates.

We can consider tilings of polygons $P$ where we tile using congruent copies of the fourteen Stomachion pieces. Since each piece in the Stomachion can be divided 
Fig. 4 The basic polygons in $(1,2)$ packings, a box and a kite
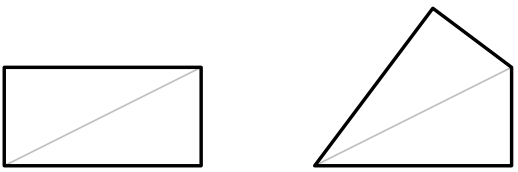

Fig. 5 A tiling of $10 \times 10$ square using one-hundred-seventy-two $(1,1)$ and four $(1,7)$ triangles

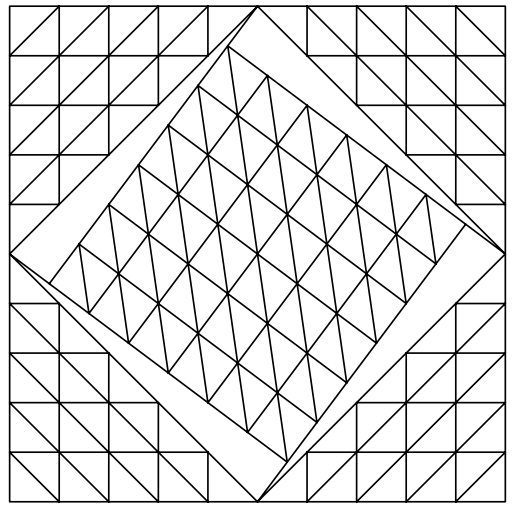

into lattice triangles with the only divisors of $a_{j}^{2}+b_{j}^{2}$ being $2,5,13,17$, we get the following by using Theorems 2 and 4 .

Corollary 5 Let $P$ be a simple polygon in the plane such that the coordinates of the vertices of $P$ are rational, and one of the vertices of $P$ is the origin with an adjacent vertex on the $x$-axis. Suppose that $P$ is dissected into congruent copies of the fourteen Stomachion pieces. Then the coordinates of the vertices are of the form $p /\left(5^{q} 13^{r} 17^{s}\right)$.

Since the Stomachion pieces include a $(2,3)$ and a $(3,6)$ triangle we see that the powers of 5 and 13 can be arbitrarily large in the denominator. However, it is unknown whether the power 17 can be arbitrarily large (or even 1).

If we examine the packing in Fig. 2 we see that all the triangles could be glued back to back on their hypotenuses. It is a simple exercise to show using Theorem 1 that for $(1,2)$ packings we will always be able to glue triangles on their hypotenuses, so in this case we are actually packing with "boxes" and "kites" (see Fig. 4).

This more generally will occur whenever the triangles $\Delta_{i}=\left(a_{i}, b_{i}\right)$ have irrational hypotenuses which are linearly independent (over $\mathbb{Q}$ ). However when we have two hypotenuses which are rational multiples of each other, or a rational hypotenuse, more interesting behavior can occur. As an example consider the tiling in Fig. 5 which uses $(1,1)$ and $(1,7)$ triangles (with hypotenuses $\sqrt{2}$ and $5 \sqrt{2}$ respectively). In this case our tiling does not consist only of boxes and kites.

We can use lattice triangles to tile polygons that do not have all rational coordinates. For example we can use two $(1,1)$ triangles and four $(1,2)$ triangles to tile $[0, \sqrt{5}] \times[0, \sqrt{5}]$. More generally, the proofs that we have given can be used to show in the case when our polygon does not have rational coordinates that the coordinates of the vertices also have some restricted forms. As an example, it can 
be shown in the tiling of $[0, \sqrt{r}] \times[0, \sqrt{r}]$ ( $r$ not a square) with right triangles $\Delta_{1}=\left(a_{1}, b_{1}\right), \ldots, \Delta_{k}=\left(a_{k}, b_{k}\right)$ the coordinates of the vertices are of the form $p \sqrt{r} / \prod_{i=1}^{k}\left(a_{i}^{2}+b_{i}^{2}\right)^{s_{i}}$.

\section{References}

1. Chung, F., Graham, R.: A tour of Archimedes' Stomachion. www.math.ucsd.edu/ fan/stomach/

2. Laczkovich, M.: Tilings of polygons with similar triangles. Combinatorica 10, 281-306 (1990)

3. Laczkovich, M.: Tilings of triangles. Discrete Math. 140, 79-94 (1995)

4. Laczkovich, M.: Tilings of polygons with similar triangles, II. Discrete Comput. Geom. 19, 411-425 (1998)

5. Su, Z., Ding, R.: Tilings of orthogonal polygons with similar rectangles or triangles. J. Appl. Math. Comput. 17, 343-350 (2005)

6. Szegedy, B.: Tilings of the square with similar right triangles. Combinatorica 21, 139-144 (2001) 